\title{
Equilibrium and nonequilibrium thermodynamics of particle-stabilized thin liquid films
}

\author{
J. Bławzdziewicz \\ Department of Mechanical Engineering, Yale University, P.O. Box 20-8286, New Haven, CT 06520 \\ E. Wajnryb \\ IPPT, Świętokrzyska 21, Warsaw, Poland
}

(Dated: November 29, 2018)

\begin{abstract}
Our recent quasi-two-dimensional thermodynamic description of thin-liquid films stabilized by colloidal particles is generalized to describe nonuniform equilibrium states of films in external potentials and nonequilibrium transport processes produced in the film by gradients of thermodynamic forces. Using a Monte-Carlo simulation method, we have determined equilibrium equations of state for a film stabilized by a suspension of hard spheres. Employing a multipolar-expansion method combined with a flow-reflection technique, we have also evaluated the short-time film-viscosity coefficients and collective particle mobility.
\end{abstract}

\section{INTRODUCTION}

Owing to the presence of oscillatory structural forces, static and dynamic properties of thin liquid films stabilized by colloidal particles, micelles, or macromolecules are quite different than the properties of particle-free films [1, 2, 3]. A striking example of thin-film behavior that is caused by oscillatory structural forces is the stepwise-thinning (i.e., film stratification) phenomenon. Stepwise thinning - or thickening [4] - occurs in liquid films stabilized by colloidal particles [5, 6, 7, 8], polyelectrolytes [9, 10], and surfactant solutions [11]. Such films undergo a sequence of stepwise transitions between regions of different, but uniform, thickness. In some cases, quite complex multiphase structures are obtained [12, 13].

The stepwise-thinning in particle-stabilized thin liquid films has been studied experimentally [3, 6, 7, 14, 15, 16 and theoretically [3, 8, 17, 18, 19, 20]. Most of the analyses of the mechanism of stepwise thinning focused on the role of normal structural force produced by the suspended particles 3 , $8,14,18,19,20]$. However, as we have pointed out in our recent paper [17], not only the normal but also lateral structural forces play an important role in the film-stratification phenomenon.

To include the lateral structural forces in our description of phase equilibria in particle-stabilized thin liquid films we have proposed a quasi-two-dimensional thermodynamic formalism 17, 21]. In this formalism the film area, film volume, and number of colloidal particles are independent parameters of state. The corresponding conjugate intensive parameters are the film tension, normal pressure, and chemical potential of the colloidal particles in the film.

The film tension involves the anisotropic part of the osmotic-pressure tensor of the suspension confined between the film interfaces. Hence, the lateral component of the structural force produced by the suspended particles contributes to mechanical equilibrium in the lateral direction (i.e., direction along the film). An analysis of the lateral structural forces is thus essential for describing film properties, in particular, for formulating the equilibrium coexistence conditions between film phases of different thickness [17].

In the present paper the ideas introduced in [17] are developed in two directions. First, we propose a theoretical description of equilibrium states of films with varying thickness $h$ and nonuniform particle number density per unit area $\tilde{n}_{\mathrm{c}}$ when lateral external forces are present. Second, we present a systematic analysis of macroscopic film dynamics, based on effective $2 \mathrm{D}$ transport equations that involve viscous dissipation and diffusive particle flux.

We also provide numerical results for equilibrium and nonequilibrium properties of a film stabilized by a suspension of hard spheres. The normal stress and film tension are obtained from the contact value of the pair distribution, which is calculated using a Monte-Carlo technique. To determine the chemical potential of the particles confined in the film we propose a new evaluation method, based on integration of the Gibbs-Duhem relation that links variations of the particle chemical potential to the corresponding variations of the normal osmotic pressure and film tension. The transport coefficients in our nonequilibrium theory are determined using a Stokesian-dynamics algorithm for a periodic system of spheres [23, 24], combined with a flow-reflection technique.

Our paper is organized as follows. A general framework for a description of nonuniform equilibrium states in particle-stabilized thin-liquid films is developed in Sec.[II. followed by an analysis of nonequilibrium transport processes in Sec. III. Short-time transport coefficients are evaluated in Sec. IV] and concluding remarks are given in Sec. V]

\section{EQUILIBRIUM DESCRIPTION}

\section{A. Particle-stabilized films}

We consider a thin liquid film of thickness $h$ and area $A$ stabilized by a colloidal suspension of hard spheres 


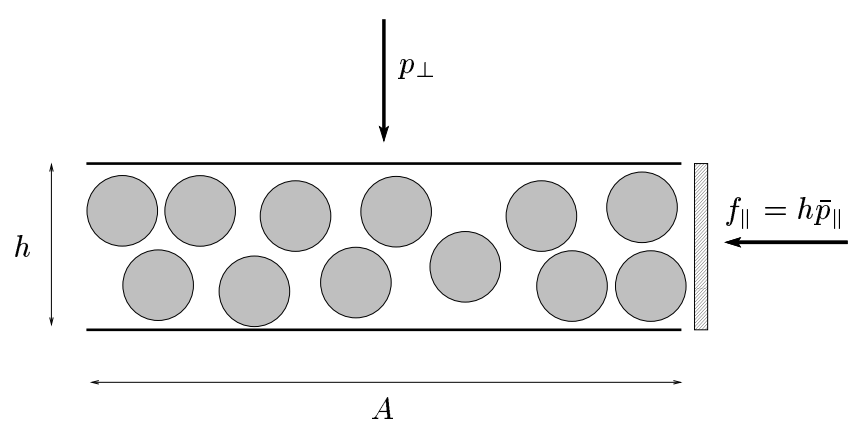

FIG. 1: Geometry of a particle-stabilized thin liquid film.

of diameter $d \sim h$. The film interfaces are surfactantfree and have a constant interfacial tension $\sigma$. The film is surrounded by an inert gas of a constant pressure $p^{(\mathrm{e})}$. The viscosity and mass density of the gas are much smaller than the corresponding parameters for the fluid in the film; therefore, the gas interacts with the film only through the static pressure $p^{(\mathrm{e})}$. The temperature $T$ in the system is uniform.

We assume that the film is approximately planar. The film thickness and the number of particles per unit area are either constant or slowly varying in the lateral directions $x$ and $y$ on the length scale $L \gg h$. Under these conditions the transverse suspension structure relaxes on the time scale $t_{\perp}$ that is much shorter than the time scale $t_{\|}$for evolution of the long-wavelength lateral modes. After the time $t_{\perp}$, the suspension in the film is thus in a local-equilibrium state, characterized by densities per unit area of conserved quantities such as the volume or number of colloidal particles.

Three-dimensional macroscopic quantities that appear in our analysis (e.g., stress and pressure tensors, suspension velocity, and particle flux) are the ensemble averages of the corresponding microscopic quantities. Assuming that the film properties are slowly varying in the lateral directions, the local area averages and ensemble averages are equivalent. In the effective quasi-two-dimensional description of the film, the averages across the film of 3D quantities are evaluated, in addition to the ensemble averages.

In all our numerical examples the suspending fluid is treated as an incompressible continuous medium. The fluid affects the thermodynamic state of the system only through the isotropic contribution to the pressure tensor and through the constant-volume constraint. The particles in the film interact via the hard-sphere potential. We note, however, that our thermodynamic considerations apply more generally, e.g., to suspensions of charged particles or small particles with finite-size hydration layers. In our hydrodynamical calculations the creeping-flow conditions are assumed, and many-body hydrodynamic interactions of the particles in the film are fully taken into account.

\section{B. Normal pressure and film tension}

Unlike the corresponding quantity in bulk suspensions, the equilibrium pressure tenor $\mathrm{p}$ in particle-stabilized films is anisotropic. This anisotropy stems from different particle ordering in the transverse and lateral directions. For films with parallel (or nearly parallel) planar interfaces, the pressure tensor has only the normal and lateral components,

$$
\mathrm{p}=p_{\perp} \hat{\mathbf{e}}_{z} \hat{\mathbf{e}}_{z}+p_{\|} \mathbf{l}_{\mathrm{s}},
$$

owing to the axial symmetry of the problem. Here $\mathrm{I}_{\mathrm{s}}=$ $\hat{\mathbf{e}}_{x} \hat{\mathbf{e}}_{x}+\hat{\mathbf{e}}_{y} \hat{\mathbf{e}}_{y}$ is the lateral unit tensor (where $\hat{\mathbf{e}}_{x}$ and $\hat{\mathbf{e}}_{y}$ are unit vectors in the lateral directions $x$ and $y$ ), and $\hat{\mathbf{e}}_{z}$ is the unit vector in the transverse direction $z$ (normal to the film).

Due to continuity of the momentum flux, the normal pressure $p_{\perp}$ is independent of the transverse coordinate $z$. However, the lateral component $p_{\|}$of the pressure tensor depends on the transverse position in the film. In our paper the average value across the film of a quantity $b$ (such as the lateral stress $p_{\|}$) will be denoted by the overbar, i.e.,

$$
\bar{b}=h^{-1} \int_{0}^{h} \mathrm{~d} z b(z) .
$$

Normal and lateral stresses acting on a section of the film are schematically illustrated in Fig. 1,

The infinitesimal work associated with the change of the film thickness $h$ and area $A$ is

$$
\mathrm{d} W=-A p_{\perp} \mathrm{d} h+\left(2 \sigma-h \bar{p}_{\|}\right) \mathrm{d} A .
$$

This expression includes the pressure and surface-tension contributions.

The quantity $f_{\|}=\left(2 \sigma-h \bar{p}_{\|}\right)$in Eq. (3) represents the lateral force (per unit length of a film section) resulting from the interfacial-tension and pressure contributions. This force, however, cannot be interpreted as film tension, and a correct relation for the film tension is given in Eq. (5). The term $\left(2 \sigma-h \bar{p}_{\|}\right) \mathrm{d} A$ in Eq. (3) describes the work associated with a change of the film area at a constant thickness $h$. The description in terms of independent variables $h$ and $A$ would give meaningless results for a film composed of an incompressible suspension, because any area change in such a system must be accompanied by the corresponding thickness change. Moreover, since we have $\bar{p}_{\|}=p_{\perp}$ for a film composed of a simple isotropic fluid, the force $f_{\|}$depends on the external pressure $p^{(\mathrm{e})}$ (which is in equilibrium with the normal pressure $p_{\perp}$ in the film). The film tension, however, should be pressure independent.

A correct film-tension representation of the equilibrium states of the film is obtained by rewriting the pressure tensor (11) in the form

$$
\mathrm{p}=p_{\perp} \mathbf{l}+\left(p_{\|}-p_{\perp}\right) \mathbf{I}_{\mathrm{s}},
$$


where $\mathrm{I}=\mathrm{I}_{\mathrm{s}}+\hat{\mathbf{e}}_{z} \hat{\mathbf{e}}_{z}$ is the $3 \mathrm{D}$ unit tensor. The first term on the right-hand side of the above relation is an isotropic pressure component that should not contribute to the film tension (as discussed above). The second term, integrated across the gap, corresponds to the excess lateral force per unit length of film section. Only this excess force contributes to the film tension $\gamma$. Combining the excess lateral pressure with the surface-tension contribution we obtain the relation for the film tension,

$$
\gamma=h\left(p_{\perp}-\bar{p}_{\|}\right)+2 \sigma
$$

and the corresponding expression for the work,

$$
\mathrm{d} W=-p_{\perp} \mathrm{d} V+\gamma \mathrm{d} A,
$$

where $V=A h$ is the film volume. The term $\gamma \mathrm{d} A$ in the above equation describes the work associated with the change of the film area at a constant film volume, in contrast to the corresponding term in Eq. (3).

In mechanical equilibrium, the normal pressure in the film equals the constant pressure of the surrounding gas,

$$
p_{\perp}=p^{(\mathrm{e})} .
$$

The excess lateral stresses have to balance as well, which implies that the film tension is position independent

$$
\gamma=\text { const, }
$$

provided that there are no external forces acting on the film.

In the above discussion we assumed that there is no direct interaction between the film interfaces. However, if there is such an interaction force (e.g., the van der Waals attraction or electrostatic repulsion), the normal pressure $p_{\perp}$ in Eqs. (3) -(77) should be replaced with $p_{\perp}^{\prime}=p_{\perp}+f_{\mathrm{n}}$, where $f_{\mathrm{n}}$ is the normal force per unit area, and $f_{\mathrm{n}}<0$ corresponds to attraction.

\section{Quasi-two-dimensional thermodynamic description}

Owing to the time scale separation $t_{\perp} \ll t_{\|}$, the relaxation of the suspension structure in the transverse direction is much faster than the relaxation of long-wavelength lateral modes. In a quasi-two-dimensional thermodynamic description [17] all details of the transverse structure of the film are thus averaged out. The film thickness $h=V / A$, however, is retained as a thermodynamic variable, because it can be controlled by varying the external pressure.

The fundamental thermodynamic relation for the free energy $F$ in the film-tension representation can be obtained from the expression for work (6) by using the standard entropy-maximization arguments [25]. Treating the suspension in the film as a two-component fluid (with the colloidal particles regarded as macromolecules) we get the fundamental relation in the free-energy representation

$$
\mathrm{d} F=-S \mathrm{~d} T-p_{\perp} \mathrm{d} V+\gamma \mathrm{d} A+\mu_{\mathrm{c}} \mathrm{d} N_{\mathrm{c}}+\mu_{\mathrm{f}} \mathrm{d} N_{\mathrm{f}},
$$

where $S$ is the entropy, and $N_{\mathrm{c}}$ and $N_{\mathrm{f}}$ denote, respectively, the number of colloidal particles and solvent molecules in the system, and $\mu_{\mathrm{c}}$ and $\mu_{\mathrm{f}}$ are the corresponding chemical potentials. According to the above relation and the free-energy-minimum principle, a particlestabilized film in thermodynamic equilibrium must satisfy the thermal equilibrium condition $T=$ const, the mechanical equilibrium conditions (77) and (8), and the chemical equilibrium condition

$$
\mu_{\mathrm{c}}=\text { const }, \quad \mu_{\mathrm{f}}=\text { const. }
$$

A direct consequence of the fundamental relation (9) is the Gibbs phase rule for coexisting regions of different thickness $h$ in stratified films in thermodynamic equilibrium. Assuming that the suspending fluid behaves as incompressible continuum medium, its degrees of freedom can be neglected (as discussed in Sec. IIE). Thus, the state of each phase is described by three densities per unit area: the excess entropy density, the film thickness $h=V / A$ and the number density of the colloidal particles $\tilde{n}_{\mathrm{c}}=N_{\mathrm{c}} / A$. There are also four equilibrium conditions corresponding to the intensive parameters $T$, $p_{\perp}, \gamma$, and $\mu_{\mathrm{c}}$. It follows that a two-phase system has $f=2$ degrees of freedom, and the maximum number of coexisting phases is $k=4$.

We note that in experiments with vertical films, up to seven coexisting phases of different thickness were observed [7]. In such systems, additional thermodynamic degrees of freedom are provided by the external gravity potential, and it is possible that not all equilibrium conditions are satisfied (due to dynamical constraints resulting in slow relaxation of certain intensive-parameter differences [17]).

\section{Nonuniform systems}

\section{External forces}

The physical meaning of film tension (5) can be further elucidated by considering nonuniform equilibrium states of a film in external lateral force fields. In Sec. III an analysis of such nonuniform film states will also help to determine the linear constitutive relations governing film dynamics.

We consider potential forces

$$
\mathbf{F}_{\mathrm{f}}=-\nabla_{\mathrm{s}} \Psi_{\mathrm{f}}, \quad \mathbf{F}_{\mathrm{c}}=-\nabla_{\mathrm{s}} \Psi_{\mathrm{c}}
$$

acting, respectively, on the fluid molecules and colloidal particles. It is assumed that the potentials $\Psi_{\mathrm{f}}$ and $\Psi_{\mathrm{c}}$ depend only on the lateral position $\boldsymbol{\rho}=x \hat{\mathbf{e}}_{x}+y \hat{\mathbf{e}}_{y}$. 
The force fields (11) are normalized per single particle. Thus, the total force density per unit area of the film is

$$
\tilde{\mathbf{f}}=\tilde{n}_{\mathrm{f}} \mathbf{F}_{\mathrm{f}}+\tilde{n}_{\mathrm{c}} \mathbf{F}_{\mathrm{c}},
$$

where $\tilde{n}_{\mathrm{f}}$ and $\tilde{n}_{\mathrm{c}}$ are the local values of the area number densities $\tilde{n}_{\mathrm{f}}=N_{\mathrm{f}} / A$ and $\tilde{n}_{\mathrm{c}}=N_{\mathrm{c}} / A$ of the fluid molecules and colloidal particles at the lateral position $\boldsymbol{\rho}$. Similarly, the total force density per unit volume is given by the relation

$$
\mathbf{f}=n_{\mathrm{f}} \mathbf{F}_{\mathrm{f}}+n_{\mathrm{c}} \mathbf{F}_{\mathrm{c}},
$$

where $n_{\mathrm{f}}$ and $n_{\mathrm{c}}$ are the local number densities per unit volume of the fluid molecules and colloidal particles at a (three-dimensional) position $\mathbf{r}$.

\section{Lateral stress balance}

The balance between the film tension and external force (12) can be obtained from the full 3D stress-balance equation

$$
\boldsymbol{\nabla} \cdot \mathrm{p}=\mathbf{f}
$$

(which is valid in the whole space). In the region inside the film, the pressure tensor $\mathrm{p}$ is given by Eq. (4); outside the film it is equal to the external pressure tensor,

$$
\mathrm{p}=p^{(\mathrm{e})} \mathrm{I}
$$

In Eq. (14), the interfacial contribution is neglected under the assumptiona that there is no spacial variation of the interfacial tension, $\sigma=$ const, and that the film thickness varies on the length scale $L \gg h$ (and thus the film curvature is negligible).

Combining relations (4) and (15) for the pressure tensor inside and outside the film, and using the normalstress boundary condition (7), we find that the divergence of the isotropic pressure component vanishes in the whole space. The integral of the remaining part with respect to the variable $z$ yields the lateral stress balance (cf. Appendix A

$$
\nabla_{\mathrm{s}} \cdot h\left(\bar{p}_{\|}-p_{\perp}\right) \mathrm{I}_{\mathrm{s}}=\tilde{\mathbf{f}}
$$

where $\tilde{\mathbf{f}}$ is the area force density (12). By using definition (5) of film tension, and applying the assumptions $L \gg h$ and $\sigma=$ const to include the interfacial-tension term, the lateral stress-balance equation (16) can be expressed as the hydrostatic condition for the film-tension gradient

$$
\nabla_{\mathrm{s}} \gamma=-\tilde{\mathbf{f}}
$$

Equation (17) requires that, in equilibrium, the area force density be a gradient of a $2 \mathrm{D}$ potential,

$$
\tilde{\mathbf{f}}=-\nabla_{\mathrm{s}} \tilde{\psi}
$$

Thus, in the presence of external force field (12), the equilibrium condition (8) is replaced by the hydrostatic condition for the film tension,

$$
\gamma-\tilde{\psi}=\text { const }
$$

A more general discussion of the lateral stress balance in the film, applicable to equilibrium and nonequilibrium states, is given in Appendix A

\section{Chemical potentials}

External forces (11) modify not only the lateral stress balance, but also the equilibrium conditions (10) for the chemical potentials of the suspending fluid and colloidal component. As in the standard thermodynamics of bulk systems, the modified chemical equilibrium conditions are

$$
\mu_{\mathrm{f}}+\Psi_{\mathrm{f}}=\text { const }, \quad \mu_{\mathrm{c}}+\Psi_{\mathrm{c}}=\text { const. }
$$

We note that the above expressions are consistent with the lateral stress balance (17), owing to the GibbsDuhem relation

$$
-S \mathrm{~d} T+V \mathrm{~d} p_{\perp}-A \mathrm{~d} \gamma-N_{\mathrm{c}} \mathrm{d} \mu_{\mathrm{c}}-N_{\mathrm{f}} \mathrm{d} \mu_{\mathrm{f}}=0
$$

(which is obtained from (9) by the usual Legendre transformation of all extensive variables). At constant $T$ and $p_{\perp}$, the Gibbs-Duhem relation yields

$$
\nabla_{\mathrm{s}} \gamma=-\tilde{n}_{\mathrm{c}} \nabla_{\mathrm{s}} \mu_{\mathrm{c}}-\tilde{n}_{\mathrm{f}} \boldsymbol{\nabla}_{\mathrm{s}} \mu_{\mathrm{f}} .
$$

By inserting the equilibrium conditions (20) into (22) and using the definition (12) of the area force density $\tilde{\mathbf{f}}$, the lateral stress-balance equation (17) is recovered.

\section{E. Incompressible suspension}

In our further considerations we focus on films composed of an incompressible suspension. It is assumed that both the solvent and the suspended particles are incompressible, and changes of the suspension volume due to interaction of particle hydration layers are negligible.

The number of solvent molecules in such a film is set by the film volume $V$ and the colloidal-particle volume fraction $\phi_{\mathrm{c}}=\frac{1}{6} \pi d^{3} \bar{n}_{\mathrm{c}}$, where $\bar{n}_{\mathrm{c}}=N_{\mathrm{c}} / V$ is the particle number density averaged over film thickness. The chemical potential $\mu_{\mathrm{f}}$ of the solvent is not an independent function of state, either. Under isothermal conditions, assumed herein, $\mu_{\mathrm{f}}$ is fully determined by the Gibbs-Duhem relation for the suspending fluid

$$
n_{\mathrm{f}}^{(0)} \mathrm{d} \mu_{\mathrm{f}}=\mathrm{d} p_{\mathrm{f}}
$$

(where $n_{\mathrm{f}}^{(0)}$ is the number density per unit volume for the fluid molecules in pure solvent). The thermodynamic 
degrees of freedom associated with the solvent variables $\mu_{\mathrm{f}}$ and $N_{\mathrm{f}}$ can thus be eliminated from the fundamental relation (9).

The thermodynamic description of an incompressible system can be rephrased in terms of appropriate excess quantities describing the colloidal contributions to thermodynamic functions of state. In this section we define excess quantities and formulate the corresponding equilibrium conditions.

The pressure tensor in the film,

$$
\mathrm{p}=p_{\mathrm{f}} \boldsymbol{l}+\mathrm{p}_{\mathrm{c}},
$$

is decomposed into the isotropic fluid pressure $p_{\mathrm{f}}$ and the osmotic pressure tensor

$$
\mathrm{p}_{\mathrm{c}}=p_{\mathrm{c} \perp} \hat{\mathbf{e}}_{z} \hat{\mathbf{e}}_{z}+p_{\mathrm{c} \|} \mathbf{l}_{\mathrm{s}}
$$

that results from the presence of the colloidal particles. The isotropic fluid pressure $p_{\mathrm{f}}$ does not contribute to film tension, according to the definition (5). It is, therefore, convenient to rewrite the film tension

$$
\gamma=\gamma_{\mathrm{c}}+2 \sigma
$$

as a sum of the interfacial tension term and the colloidal contribution

$$
\gamma_{\mathrm{c}}=h\left(p_{\mathrm{c} \perp}-\bar{p}_{\mathrm{c} \|}\right)
$$

Since we assume no variation of the interfacial tension along the film, the colloidal contribution $\gamma_{\mathrm{c}}$ differs from the full film tension $\gamma$ only by a constant. Thus, the hydrostatic-equilibrium condition for film tension (19) remains, essentially, unchanged,

$$
\gamma_{\mathrm{c}}-\tilde{\psi}=\text { const. }
$$

In a system with no external potentials, the fluid pressure satisfies the equilibrium condition $p_{\mathrm{f}}=$ const. In the presence of an external potential (11) equilibrium condition (20a) and the Gibbs-Duhem relation (23) yield

$$
p_{\mathrm{f}}+\psi_{\mathrm{f}}=\mathrm{const}
$$

where

$$
\psi_{\mathrm{f}}=n_{\mathrm{f}}^{(0)} \Psi_{\mathrm{f}}
$$

is the potential of the volume force acting on the fluid phase. Equation (29) represents the usual hydrostaticequilibrium condition for the suspending fluid.

With the help of the hydrostatic relation (29), the constant-normal-pressure condition (7) can be transformed into the corresponding equilibrium condition for the osmotic normal pressure $p_{\mathrm{c} \perp}$,

$$
p_{\mathrm{c} \perp}-\psi_{\mathrm{f}}=\text { const. }
$$

The equilibrium stress-balance equation (31) relates the normal osmotic pressure in the film to the potential $\psi_{\mathrm{f}}$ of the lateral force acting on the fluid. Thus, in equilibrium states with a nonzero lateral force, the normal osmotic pressure varies along the film.

The remaining equilibrium condition that has to be considered, is relation (20 b) for the chemical potential of the colloidal particles $\mu_{\mathrm{c}}$. In the fundamental relation (9), the term $\mu_{\mathrm{c}} \mathrm{d} N_{\mathrm{c}}$ corresponds to the change of the free energy $F$ when a colloidal particle is added to the system at a fixed volume and number of fluid particles. However, in an incompressible suspension, a particle that is moved from one subsystem of the film to another is always replaced by the equivalent amount of the suspending fluid. Accordingly, the quantity $\mu_{\mathrm{c}}$ involves not only the configurational contribution resulting from the distribution of the colloidal particles, but also an additional part corresponding to the amount of the fluid displaced by a particle.

To define the excess chemical potential $\mu_{\mathrm{c}}^{\prime}$ that is free of the fluid contribution, we begin with the Gibbs-Duhem relation (21). By combining (21) with the Gibbs-Duhem relation for the suspending fluid (23) we obtain the identity

$$
V \mathrm{~d} p_{\perp}-A \mathrm{~d} \gamma-N_{\mathrm{c}} \mathrm{d} \mu_{\mathrm{c}}-\left(1-\phi_{\mathrm{c}}\right) V \mathrm{~d} p_{\mathrm{f}}=0,
$$

where the constant-temperature conditions are assumed. Defining the excess chemical potential of the colloidal particles as

$$
\mu_{\mathrm{c}}^{\prime}=\mu_{\mathrm{c}}-v_{\mathrm{p}} p_{\mathrm{f}}
$$

(where $v_{\mathrm{p}}$ is the volume of a colloidal particle), and using relation $p_{\perp}=p_{\mathrm{c} \perp}+p_{\mathrm{f}}$, we find the Gibbs-Duhem relation for $\mu_{\mathrm{c}}^{\prime}$,

$$
\mathrm{d} \mu_{\mathrm{c}}^{\prime}=\bar{n}_{\mathrm{c}}^{-1} \mathrm{~d} p_{\mathrm{c} \perp}-\tilde{n}_{\mathrm{c}}^{-1} \mathrm{~d} \gamma_{\mathrm{c}} .
$$

The above equation indicates that the quantity $\mu_{\mathrm{c}}^{\prime}$ is determined (up to a temperature-dependent additive constant) by the osmotic normal pressure and film tension, which justifies our interpretation of $\mu_{\mathrm{c}}^{\prime}$ as the excess chemical potential.

In order to rephrase the chemical equilibrium condition for the colloidal particles in terms of the excess chemical potential $\mu_{\mathrm{c}}^{\prime}$, relations (29) and (20) are inserted into (33). As the result we get the chemical equilibrium condition for the excess quantities

$$
\mu_{\mathrm{c}}^{\prime}+\Psi_{\mathrm{c}}^{\prime}=\text { const },
$$

where the excess potential

$$
\Psi_{\mathrm{c}}^{\prime}=\Psi_{\mathrm{c}}-v_{\mathrm{p}} \psi_{\mathrm{f}}
$$

of the external force acting on the colloidal particles includes the buoyancy contribution resulting from the pressure gradient in the suspending fluid. 


\section{F. Numerical results}

\section{Evaluation technique}

In this section we present some numerical results for the osmotic-pressure tensor $\mathrm{p}_{\mathrm{c}}$, film tension $\gamma_{\mathrm{c}}$, and particle chemical potential $\mu_{\mathrm{c}}^{\prime}$ for a film stabilized by a suspension of hard spheres of diameter $d$. A standard Metropolis Monte-Carlo algorithm was used to generate equilibrium ensemble for a system of a hundred hard spheres in a film with periodic boundary conditions in the lateral directions. The results were obtained for several volume fractions of colloidal particles $\phi_{\mathrm{c}}$, as a function of film thickness $h$.

a. Osmotic-pressure tensor The ensemble-averaged osmotic pressure tensor has been evaluated using a hardsphere generalization of the Kirkwood-Buff expression for the pressure tensor in an inhomogeneous fluid [26]. Accordingly, the colloidal contribution to the pressure tensor $\mathrm{p}_{\mathrm{c}}$, averaged over the space $\hat{V}=A(h-d)$ accessible to the particle centers, is obtained from the relation

$$
\frac{\hat{\mathrm{p}}_{\mathrm{c}}}{k T}=\hat{n}_{\mathrm{c}}+\frac{1}{2} d^{3}\left\langle\int \hat{\mathbf{r}} \hat{\mathbf{r}} n_{2}^{\mathrm{eq}}\left(\mathbf{r}_{1}, \mathbf{r}_{1}-d \hat{\mathbf{r}}\right) \mathrm{d}^{2} \hat{r}\right\rangle_{\hat{V}} .
$$

Here $n_{2}^{\mathrm{eq}}\left(\mathbf{r}_{1}, \mathbf{r}_{2}\right)$ is the two-particle equilibrium reduced distribution, $\hat{\mathbf{r}}=\mathbf{r} / r$ is the unit vector along the line passing through the particle centers, the integration is over the contact configurations, $\langle\cdots\rangle_{\hat{V}}$ denotes the volume average over the region $\hat{V}$, and $\hat{n}_{\mathrm{c}}=N_{\mathrm{c}} / \hat{V}$ is the corresponding particle number density.

Equation (37) generalizes a well-known expression for the pressure in a bulk hard-sphere system in terms of the contact value of the radial distribution. The contactvalue relation (37) can be derived either from the collisional contribution to the momentum flux in a hardsphere system, or by passing to the hard-sphere limit in the Kirkwood-Buff expression [26] for the pressure tensor in an inhomogeneous fluid.

In our numerical calculations, the average (37) is evaluated from a sum of contributions originating from pairs of particles separated by a distance $r_{i j}<d(1+\epsilon)$, where $\epsilon \ll 1$. The results for finite values of $\epsilon$ are extrapolated to obtain the limiting result for $\epsilon \rightarrow 0$.

The contact-value relation (37) provides a convenient basis for numerical evaluation of the pressure tensor in the film. However, $\hat{p}_{\mathrm{c}}$ involves only averaging over the accessible volume $\hat{V}$, and does not incorporate the momentum transfer from the particle centers to the wall. To obtain the pressure tensor $\bar{p}_{\mathrm{c}}$ averaged over the whole volume of the film $V$, we need to consider this momentum transfer.

The normal momentum transfer occurs when a particle collides with the interface [27]. Thus, there is a constant normal momentum flux in the geometrically excluded regions of thickness $a=\frac{1}{2} d$ next to each interface. It follows that the normal component of the pressure is not affected by the choice of the region $(V$ or $\hat{V})$ over which the average is performed. In contrast there is no lateral momentum transport in the excluded layers next to each wall, and the averages over the volumes $V$ and $\hat{V}$ differ by the factor $\hat{V} / V=(h-d) / h$.

The above arguments imply that the normal and lateral components of the tensor $\mathrm{p}_{\mathrm{c}}$ averaged over the volume $V$ are

$$
p_{\mathrm{c} \perp}=\hat{p}_{\mathrm{c} \perp}, \quad \bar{p}_{\mathrm{c} \|}=h^{-1}(h-d) \hat{p}_{\mathrm{c} \|},
$$

where $\hat{\mathrm{p}}_{\mathrm{c}}=\hat{p}_{\mathrm{c} \perp} \hat{\mathbf{e}}_{z} \hat{\mathbf{e}}_{z}+\hat{p}_{\mathrm{c} \|} \mathbf{I}_{\mathrm{s}}$ is given by the contact-value relation (37). The components (38) are used to determine the colloidal contribution to film tension (27).

b. Chemical potential Our quasi-two-dimensional thermodynamic formalism can be conveniently used for evaluation of the chemical potential of the colloidal particles in the film $\mu_{\mathrm{c}}^{\prime}$ from the results for the normal pressure and film tension. Our method relies on numerical integration of the Gibbs-Duhem relation (34), using results of Monte-Carlo simulations at different values of particle volume fraction $\phi_{\mathrm{c}}$ and film thickness $h$.

For convenience, in our calculations we use a modified Gibbs-Duhem relation based directly on the pressure tensor (37), without introducing the averages (38) over the full film volume $V$. In this modified formulation, the Gibbs-Duhem relation (34) is replaced with

$$
\mathrm{d} \mu_{\mathrm{c}}^{\prime}=\hat{n}_{\mathrm{c}}^{-1} \mathrm{~d} p_{\mathrm{c} \perp}-\tilde{n}_{\mathrm{c}}^{-1} \mathrm{~d} \hat{\gamma}_{\mathrm{c}}
$$

where the modified film tension $\hat{\gamma}_{\mathrm{c}}$ is given by the formula

$$
\hat{\gamma}_{\mathrm{c}}=(h-d)\left(p_{\mathrm{c} \perp}-\hat{p}_{\mathrm{c} \|}\right),
$$

instead of 277).

We note that equations (34) and (39) are equivalent, which follows from the identities

$$
\begin{gathered}
\gamma_{\mathrm{c}}=\hat{\gamma}_{\mathrm{c}}+d p_{\mathrm{c} \perp}, \\
\bar{n}_{\mathrm{c}}^{-1}=\hat{n}_{\mathrm{c}}^{-1}+d \tilde{n}_{\mathrm{c}}^{-1},
\end{gathered}
$$

where, as previously, the overbar and the hat indicate the average particle density in the volumes $V$ and $\hat{V}$, respectively, and the tilde indicates the particle density per unit area.

We have verified that numerical integration of GibbsDuhem relation (39) over different paths in the thermodynamic space of states yields equivalent results. To obtain proper normalization of the chemical potential, relation (39) is integrated from a low-density state, where we can use the perfect-gas result for the chemical potential.

Since equilibrium properties of a confined suspension do not depend on the details of the dynamic boundary conditions at the interfaces of the film (e.g., free interface or rigid wall), our method for evaluating the chemical potential can be applied not only to thin liquid films, but also to other systems with a slit-pore geometry. In particular, our method can be used to determine the chemical potential of spherical macromolecules in the gap between two much larger particles in a system where depletion interactions are important. Our method can also be generalized to systems interacting via continuous or anisotropic potentials. 

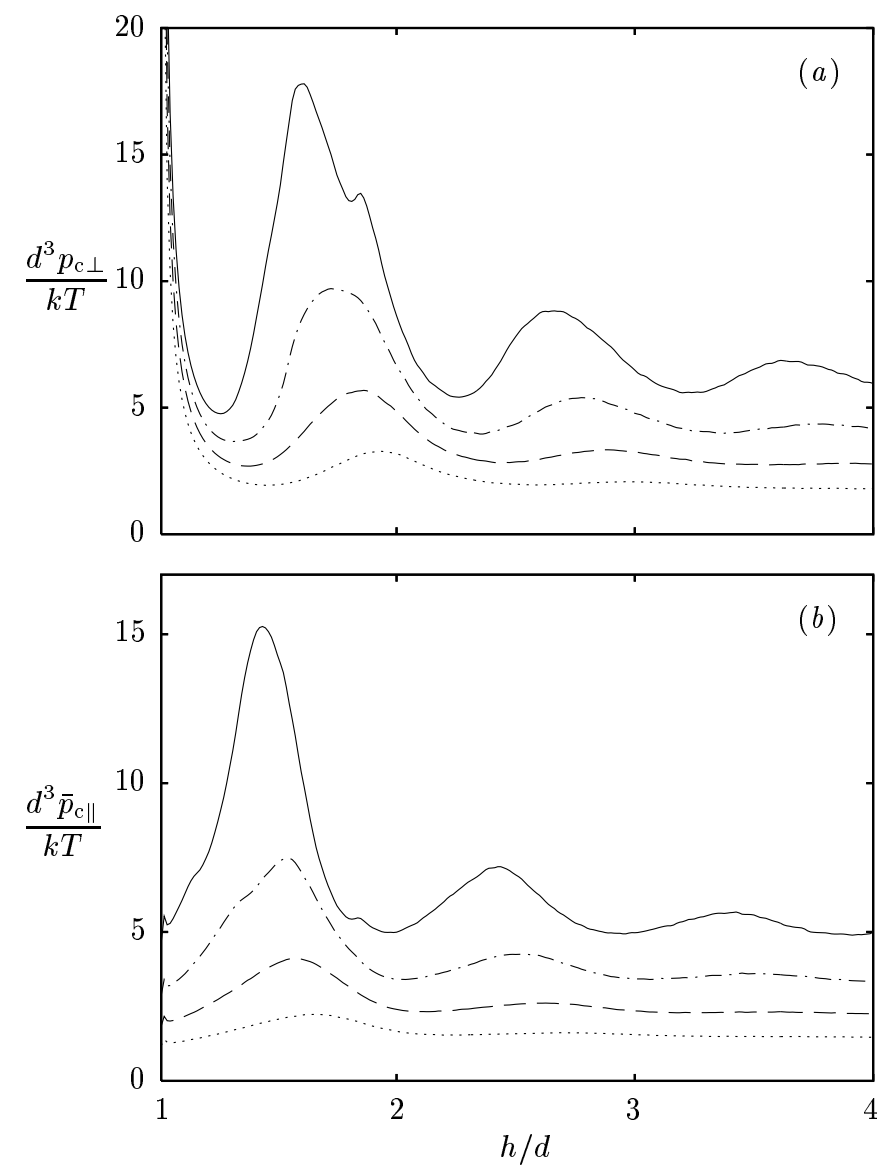

FIG. 2: (a) Normal and (b) lateral osmotic pressure in the film, versus film thickness $h$, for particle volume fractions $\phi_{\mathrm{c}}=$ 0.25 (dotted line), 0.3 (dashed), 0.35 (dot-dashed), and 0.4 (solid).

\section{Results}

The dependence of the normal and lateral pressure components (38) on the film thickness is illustrated in Fig. 2, which shows $p_{\mathrm{c} \perp}$ and $\bar{p}_{\mathrm{c} \|}$ versus $h$ for several values of the particle volume fraction $\phi_{\mathrm{c}}$. The corresponding behavior of the film tension $\gamma_{\mathrm{c}}$ and excess chemical potential $\mu_{\mathrm{c}}^{\prime}$ is depicted in Figs. 3 and 4 [28].

The results plotted in Fig. 22 indicate that both $p_{\mathrm{c} \perp}$ and $\bar{p}_{\mathrm{c} \|}$ are oscillatory functions of $h$. However, the phase of the oscillations of the normal and lateral pressure components significantly differ, with the maxima of $\bar{p}_{\mathrm{c} \|}$ occurring at smaller values of $h$ than the maxima of $p_{\mathrm{c} \perp}$. Film tension (27) and chemical potential $\mu_{\mathrm{c}}^{\prime}$ [which is obtained from the pressure tensor by integrating the GibbsDuhem relation (39)] are also oscillatory functions of $h$. (cf., Figs. 3 and 4).

The oscillatory behavior of the pressure tensor is typical of effective structural interactions in confined domains. The nonmonotonic dependence on $h$ stems from the short-range layered ordering of the suspension in the film [29]. A qualitative understanding of the mechanism

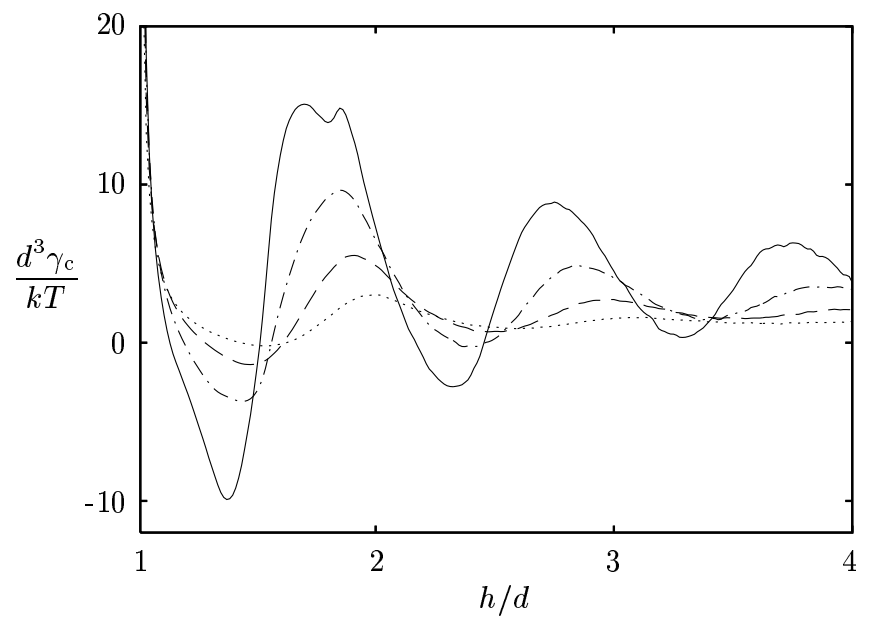

FIG. 3: Particle contribution to film tension, versus film thickness $h$, for the same values of volume fraction $\phi_{\mathrm{c}}$ as in Fig. 2.

leading to the pressure oscillations and of the source of the shift between the maxima of $p_{\mathrm{c} \perp}$ and $\bar{p}_{\mathrm{c} \|}$ can be gained from an analysis of the suspension structure for different values of the film thickness $h$. In Fig. 5 we show the local particle density $n_{\mathrm{c}}$ in the film for two values of interface separation, corresponding to the maximum $(h / d=1.6)$ and minimum $(h / d=2.25)$ of the normal pressure $p_{\mathrm{c} \perp}$ [cf., Fig. 2( $(a)$ ].

The results shown in Fig. 5 indicate that for both values of $h / d$, there are two particle layers in the film. For $h / d=1.6$, the two layers barely fit into the available space $\hat{V}$; particle concentration is thus strongly focused in thin regions adjacent to each interface, and the suspension exerts high normal pressure $p_{\mathrm{c} \perp}$ on the interfaces of the film. For $h / d=2.25$, the particle layers are more

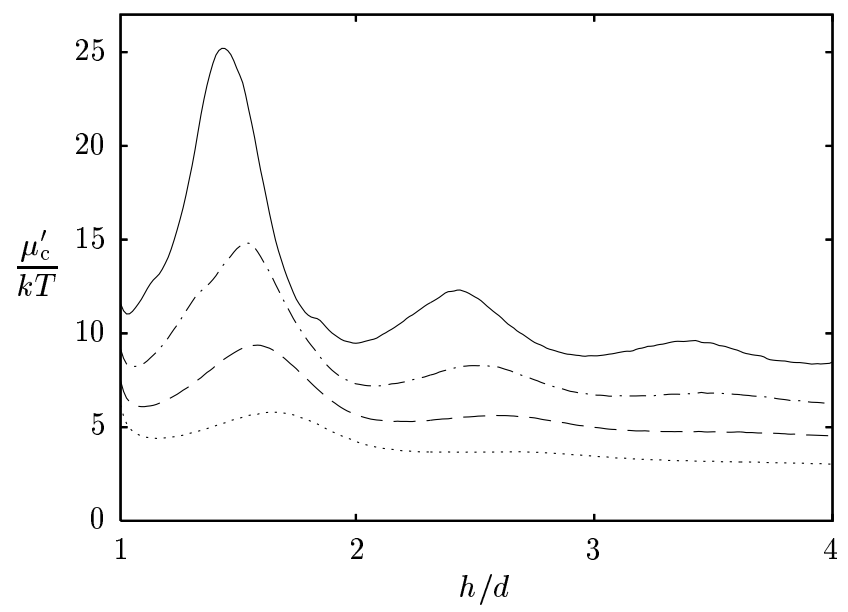

FIG. 4: Excess chemical potential of the particles in the film, versus film thickness $h$, for the same values of volume fraction $\phi_{\mathrm{c}}$ as in Fig. 2 The normalization corresponds to the asymptotic behavior $\mu_{\mathrm{c}}^{\prime}=k T \ln \left(d^{3} \hat{n}_{\mathrm{c}}\right)$ for $\phi_{\mathrm{c}} \ll 1$. 


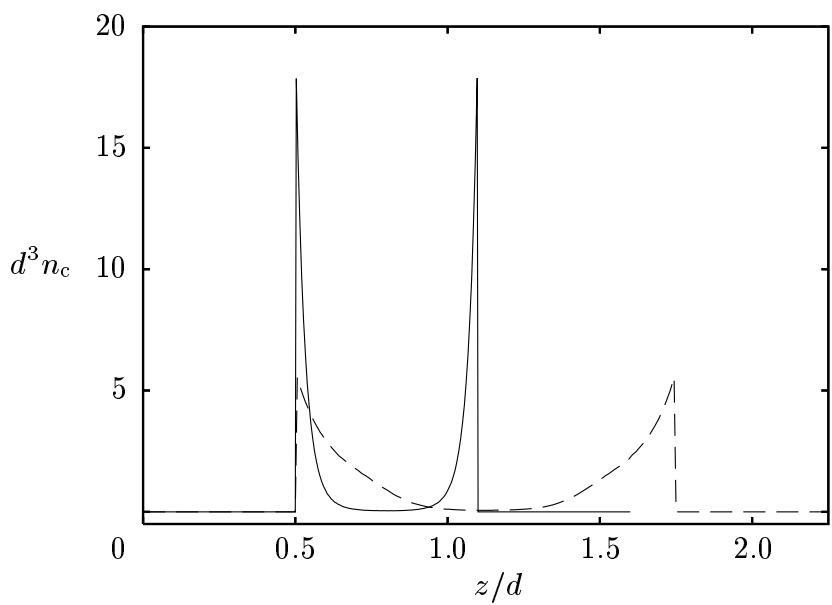

FIG. 5: Local particle number density $n_{\mathrm{c}}$ (per unit volume), versus position $z$ across the film, for volume fraction $\phi_{\mathrm{c}}=0.4$ and two values of film thickness $h / d=1.6$ (solid line) and $h / d=2.25$ (dashed line). Relative to the film thickness, the particle distribution is much narrower for $h / d=1.6$ (maximum of $p_{\mathrm{c} \perp}$ in Fig. 2) than the distribution for $h / d=2.25$ (minimum of $p_{\mathrm{c} \perp}$ ).

diffuse, and the normal pressure is much lower.

In contrast to the behavior of $p_{\mathrm{c} \perp}$, the lateral pressure component $\bar{p}_{\mathrm{c} \|}$ remains moderate both for $h / d=1.6$ and $h / d=2.25$ [cf. the results shown in Fig. $2(b)$ ]. This is because for $h / d=1.6$ there are very few particles in the central portion of the film, which implies that there is no lateral momentum transport in this region. The average value thus remains relatively low, in spite of high momentum flux in the layers of high particle concentration. As a result of this mechanism, the maxima of the lateral pressure $p_{\mathrm{c} \|}$ (averaged across the film) occur for interface separations where both, the local lateral momentum transport is relatively large, and it takes place over sufficiently broad regions.

The oscillatory form of the intensive thermodynamic parameters $p_{\mathrm{c} \perp}, \gamma_{\mathrm{c}}$, and $\mu_{\mathrm{c}}^{\prime}$ strongly affects properties of particle-stabilized films. First, owing to these oscillations, the coexistence between uniform film phases of different thickness is possible, as observed experimentally [5, 6, 7, 8] and discussed in our recent paper [17].

Next, a small perturbation $\delta h$ of the film thickness from a uniform stable equilibrium state produces restoring force proportional to the lateral gradient of $\delta h$. In contrast, in particle-free films the leading-order restoring force results from the capillary pressure, and it is thus proportional to the gradient of the film curvature $k \sim \nabla_{\mathrm{s}}^{2} h$. For long-wavelength perturbations, the restoring force acting in particle-stabilized films is thus significantly stronger than the restoring force in particlefree films. Therefore, particle-stabilized films often retain nearly uniform thickness during the drainage process (except for stepwise changes associated with phase transitions), whereas particle-free films usually assume a dimpled shape with nearly constant curvature.

\section{MACROSCOPIC TRANSPORT PROCESSES}

After discussing equilibrium thermodynamics of particle-stabilized thin liquid films, we now focus on linear transport processes that occur when such films are perturbed from equilibrium. In Sec. ЏII we present quasi-two-dimensional continuity equations for conserved parameters characterizing local-equilibrium states of the film, and in Secs. IIIB and III C we formulate constitutive relations between thermodynamic forces and macroscopic fluxes in the conservation equations.

\section{A. Conservation equations}

The thermodynamic analysis presented in Sec. III indicates that a particle-stabilized thin liquid film behaves as a two-component 2D compressible fluid, even though the suspension in the film is incompressible. The film thickness plays the role of the mass density, per unit area, and the number of colloidal particles per unit area corresponds to the concentration of the solute.

In the quasi-two-dimensional description, the macroscopic motion of the suspension in the film is described by the lateral velocity

$$
\mathbf{v}_{\mathrm{s}}=\mathrm{I}_{\mathrm{s}} \cdot \overline{\mathbf{v}},
$$

where $\mathbf{v}$ denotes the ensemble-averaged velocity field, and the overbar denotes the average across the film (2). The macroscopic motion of the particles in the film is characterized by the $2 \mathrm{D}$ lateral particle flux

$$
\tilde{\mathbf{j}}_{\mathrm{c}}=\mathrm{I}_{\mathrm{s}} \cdot h \overline{\mathbf{j}}_{\mathrm{c}},
$$

where $\overline{\mathbf{j}}_{\mathrm{c}}$ is the $3 \mathrm{D}$ particle flux $\mathbf{j}_{\mathrm{c}}$ averaged across the film. We use the normalization where the particle flux $\tilde{\mathbf{j}}_{\mathrm{c}}$ is measured with respect to the local suspension velocity $\mathbf{v}_{\mathrm{s}}$.

Definition (43) and the incompressibility condition $\boldsymbol{\nabla} \cdot \mathbf{v}=0$ for the 3D flow field yield the 2D continuity equation for the film thickness $h$,

$$
\frac{\partial h}{\partial t}=-\nabla_{\mathbf{s}} \cdot\left(h \mathbf{v}_{\mathbf{s}}\right),
$$

where $t$ denotes time. Similarly, the evolution of the particle density per unit area is governed by the $2 \mathrm{D}$ continuity equation

$$
\frac{\partial \tilde{n}_{\mathrm{c}}}{\partial t}=-\boldsymbol{\nabla}_{\mathrm{s}} \cdot\left(\tilde{n}_{\mathrm{c}} \mathbf{v}_{\mathrm{s}}+\tilde{\mathbf{j}}_{\mathrm{c}}\right) .
$$

To obtain a full description of the film dynamics, the continuity relations (45) and (46) have to be supplemented with the stress-balance equations and the constitutive equations for the stress tensor and the particle flux in the film. 


\section{B. Stress-balance equations}

\section{Lateral stress balance}

According to the results given in Appendix A, the stress-balance equation in the film-tension representation involves the effective $2 \mathrm{D}$ surface stress $\tilde{\tau}$ and area-force density (12),

$$
\nabla_{\mathrm{s}} \cdot \tilde{\tau}=-\tilde{\mathbf{f}} .
$$

The effective surface stress, defined by relations (A7) and (A13), equals the excess stress beyond the constant isotropic background pressure $p^{(\mathrm{e})}$ I, integrated across the film. As in all our dynamic considerations, in the stressbalance equation (47), creeping-flow conditions are assumed.

In equilibrium, the $2 \mathrm{D}$ surface-stress tensor reduces to the isotropic film tension, $\tilde{\tau}=\gamma \mathbf{l}_{\mathrm{s}}$. In a nonequilibrium state, the surface stress

$$
\tilde{\tau}=\gamma \mathrm{l}_{\mathrm{s}}+\tilde{\boldsymbol{\tau}}^{\prime}
$$

is a sum of the local-equilibrium contribution $\gamma \mathbf{I}_{\mathrm{s}}$ and the viscous part $\tilde{\boldsymbol{\tau}}^{\prime}$. In the above equation, the local value of the film tension $\gamma$ is given by the equilibrium equation of state, consistent with the standard local-equilibrium assumption [30]. For a particle-stabilized film, the film tension $\gamma=\gamma\left(h, \tilde{n}_{\mathrm{c}}\right)$ is fully determined by the local film thickness $h$ and local number of colloidal particles per unit area of the film $\tilde{n}_{\mathrm{c}}$.

In our further discussion, we assume the linearresponse regime and long-wavelength limit. Under these conditions, the viscous stress $\tilde{\boldsymbol{\tau}}^{\prime}$ is related to the lateral velocity (43) via two film-viscosity coefficients: the shear and the expansion viscosities $\tilde{\eta}$ and $\tilde{\kappa}$. Accordingly, we have

$$
\tilde{\boldsymbol{\tau}}^{\prime}=2 \tilde{\eta}\left[\boldsymbol{\nabla}_{\mathrm{s}} \mathbf{v}_{\mathrm{s}}\right]_{\mathrm{d}}+\tilde{\kappa}\left(\boldsymbol{\nabla}_{\mathrm{s}} \cdot \mathbf{v}_{\mathrm{s}}\right) \mathbf{I}_{\mathrm{s}}
$$

where

$$
\left[\boldsymbol{\nabla}_{\mathrm{s}} \mathbf{v}_{\mathrm{s}}\right]_{\mathrm{d}}=\frac{1}{2}\left[\boldsymbol{\nabla}_{\mathrm{s}} \mathbf{v}_{\mathrm{s}}+\left(\boldsymbol{\nabla}_{\mathrm{s}} \mathbf{v}_{\mathrm{s}}\right)^{\dagger}-\boldsymbol{\nabla}_{\mathrm{s}} \cdot \mathbf{v}_{\mathrm{s}} \mathrm{l}_{\mathrm{s}}\right]
$$

is the deviatoric part of the lateral strain-rate tensor $\nabla_{\mathrm{s}} \mathbf{v}_{\mathrm{s}}$, with the dagger denoting the transpose.

\section{Normal stress balance}

Owing to the suspension incompressibility, the normal balance of the total stress in the film does not have an independent dynamical meaning, unlike the lateral balance (47). However, the normal osmotic pressure component is an important dynamic quantity, because it drives a lateral particle flux. These results are derived below.

In a nonequilibrium state of the film, the equilibrium condition (77) is replaced by the normal-stress balance

$$
\tau_{\perp}=-p^{(e)},
$$

where $\tau_{\perp}$ is the normal component of the stress tensor $\tau$. Unlike the effective surface stress (48), the normal stress $\tau_{\perp}$ cannot be uniquely decomposed into the equilibrium and nonequilibrium components. This is because in an incompressible suspension the isotropic component of the pressure tensor (and thus also the normal pressure $p_{\perp}$ ) is not a well-defined function of the densities of the conserved quantities $h=V / A$ and $\tilde{n}_{\mathrm{c}}=N_{\mathrm{c}} / A$. There is thus no independent constitutive relation for the nonequilibrium part of $\tau_{\perp}$, and any unbalanced normal stress is immediately compensated for by a proper redistribution of the isotropic component of the 3D pressure tensor. We note that such redistribution does not modify the lateral stress balance in the film, because the surface stress $\tilde{\tau}$ involves only differences between the normal and lateral components of the 3D stress, according to Eq. (A13).

While the normal stress cannot be uniquely decomposed into the equilibrium and nonequilibrium parts, there exists another useful decomposition. Namely, the stress $\tau_{\perp}$ can be decomposed into the equilibrium osmotic normal pressure $p_{\mathrm{c} \perp}$ and the remaining nonequilibrium part $\tau_{\perp}^{\prime \prime}$

$$
\tau_{\perp}=p_{\mathrm{c} \perp}+\tau_{\perp}^{\prime \prime} .
$$

The nonequilibrium stress component $\tau_{\perp}^{\prime \prime}$ involves the $3 \mathrm{D}$ isotropic fluid-pressure, and anisotropic contributions that result from $(a)$ the viscous fluid flow and (b) deviation of the particle distribution from equilibrium. Owing to the equilibrium condition (51), for given $p^{(\mathrm{e})}$ the stress $\tau_{\perp}^{\prime \prime}$ is entirely determined by the osmotic normal pressure $p_{\mathrm{c} \perp}$, which is a well-defined function of $h$ and $\tilde{n}_{\mathrm{c}}$.

\section{Particle flux}

According to the principles of the thermodynamics of irreversible processes [30], in the linear-response regime the diffusive flux of colloidal particles is driven by gradients of scalar intensive thermodynamic parameters that characterize the thermodynamic equilibrium conditions. In a particle-stabilized thin liquid film, the relevant intensive parameters are the normal osmotic pressure $p_{\mathrm{c} \perp}$ (as discussed above) and the chemical potential of the suspended particles $\mu_{\mathrm{c}}^{\prime}$. If a particle-stabilized film is subject to external lateral forces, we also have thermodynamic forces associated with the external potentials $\psi_{\mathrm{f}}$ and $\Psi_{\mathrm{c}}^{\prime}$, according to the equilibrium conditions (31) and (35). However, the spatial variations of the film tension $\gamma$ (playing a role analogous to the isotropic equilibrium pressure in simple fluid) produce only the macroscopic motion of the film. Hence, in the linear-response regime, we assume the following constitutive relation for the $2 \mathrm{D}$ diffusive particle flux

$$
\tilde{\mathbf{j}}_{\mathrm{c}}=-\lambda_{\mu} \boldsymbol{\nabla}_{\mathrm{s}}\left(\mu_{\mathrm{c}}^{\prime}+\Psi_{\mathrm{c}}^{\prime}\right)-\lambda_{\mathrm{p}} \boldsymbol{\nabla}_{\mathrm{s}}\left(p_{\mathrm{c} \perp}-\psi_{\mathrm{f}}\right) .
$$

Using the Gibbs-Duhem relation (34) it can be shown that the kinetic coefficients $\lambda_{\mu}$ and $\lambda_{\mathrm{p}}$ cannot be determined separately. Therefore, we combine them into a 
single mobility coefficient $\nu^{\prime}$. We first consider a quiescent film with no viscous stress (49). In this case the variations of the osmotic contribution to film tension $\gamma_{\mathrm{c}}$ are related to the external potentials $\Psi_{\mathrm{c}}^{\prime}$ and $\psi_{\mathrm{f}}$ via the hydrostatic condition

$$
\nabla_{\mathrm{s}} \gamma_{\mathrm{c}}=\tilde{n}_{\mathrm{c}} \boldsymbol{\nabla}_{\mathrm{s}} \Psi_{\mathrm{c}}^{\prime}+h \boldsymbol{\nabla}_{\mathrm{s}} \psi_{\mathrm{f}},
$$

which follows from Eqs. (28), (30), and (36). From the Gibbs-Duhem relation (34) we also have

$$
\nabla_{\mathrm{s}} \gamma_{\mathrm{c}}=\tilde{n}_{\mathrm{c}} \boldsymbol{\nabla}_{\mathrm{s}} \mu_{\mathrm{c}}^{\prime}-h \boldsymbol{\nabla}_{\mathrm{s}} p_{\mathrm{c} \perp} .
$$

Combining Eqs. (53) and (54) yields one-to-one correspondence between the thermodynamic forces on the right-hand side of the constitutive relation (53),

$$
\bar{n}_{\mathrm{c}} \nabla_{\mathrm{s}}\left(\mu_{\mathrm{c}}^{\prime}+\Psi_{\mathrm{c}}^{\prime}\right)=\nabla_{\mathrm{s}}\left(p_{\mathrm{c} \perp}-\psi_{\mathrm{f}}\right) .
$$

Using (56) to eliminate the osmotic normal pressure $p_{\mathrm{c} \perp}$ and potential $\psi_{\mathrm{f}}$ from Eq. (53), we find

$$
\tilde{\mathbf{j}}_{\mathrm{c}}=-\tilde{n}_{\mathrm{c}} \nu^{\prime} \nabla_{\mathrm{s}}\left(\mu_{\mathrm{c}}^{\prime}+\Psi_{\mathrm{c}}^{\prime}\right),
$$

where

$$
\nu^{\prime}=\tilde{n}_{\mathrm{c}}^{-1} \lambda_{\mu}+h^{-1} \lambda_{\mathrm{p}}
$$

is the collective mobility of the colloidal particles in the film. We note that Eq. (57) relates the particle flux $\tilde{\mathbf{j}}_{c}$ to the excess quantities (33) and (36), indicated by the prime in our notation for the mobility coefficient (58) (cf. Sec. IV A 3).

In nonequilibrium states with nonvanishing gradients of the film velocity $\mathbf{v}_{\mathrm{s}}$, the balance equation for the lateral stress involves the viscous contribution $\nabla_{\mathrm{S}} \cdot \tilde{\boldsymbol{\tau}}^{\prime}$, according to equations (47) and (48). As a result, a modified relation (56) between the thermodynamic forces in Eq. (53) includes a $\boldsymbol{\nabla}_{\mathrm{s}} \cdot \tilde{\boldsymbol{\tau}}^{\prime}$ correction. This correction, however, is small in the long-wavelength regime, because it depends on second derivatives of the film velocity $\mathbf{v}_{\mathbf{s}}$, according to the constitutive relation for the stress (49). It follows that in the linear-response and long-wavelength limit, particle transport in the film can be described using the constitutive equation (57) with a single mobility coefficient $\nu^{\prime}$.

\section{EVALUATION OF THE SHORT-TIME TRANSPORT COEFFICIENTS}

\section{A. Elementary hydrodynamic processes}

In this section we evaluate the effective film-viscosity coefficients $\tilde{\eta}$ and $\tilde{\kappa}$ and the collective particle mobility. The film interfaces are assumed to be surfactant free, so free-interface boundary conditions are assumed.

We focus on the short-time transport coefficients corresponding to the initial system response to a startup of a flow or a sudden application of an external force. The short-time transport coefficients also describe the linear response to high-frequency oscillatory forcing.

In the short-time (or high-frequency) regime the characteristic timescale of the forcing is much shorter than the structural relaxation time of the suspension in the film. Thus the deviation of the particle distribution from equilibrium is insignificant, and the Brownian contribution to the transport coefficients can be neglected. Accordingly, evaluation of the transport coefficients requires solving appropriate many-particle hydrodynamic problems for the equilibrium particle distribution of spheres in the film under creeping-flow conditions.

\section{Shear viscosity of the film}

To evaluate the effective shear viscosity coefficient $\tilde{\eta}$, we consider a film undergoing the planar straining flow with the strain-rate tensor

$$
E=\frac{1}{2}\left[\nabla \mathbf{v}+(\nabla \mathbf{v})^{\dagger}\right]
$$

given by

$$
E=\left[\begin{array}{rrr}
1 & 0 & 0 \\
0 & -1 & 0 \\
0 & 0 & 0
\end{array}\right]
$$

where the coordinates $x$ and $y$ are in the directions along the film. By the axial symmetry and translational invariance with respect to lateral directions, the corresponding ensemble-averaged $3 \mathrm{D}$ viscous-stress tensor has the form

$$
\boldsymbol{\tau}^{\prime}=2 \eta_{\|}(z) \mathrm{E},
$$

where the mesoscopic viscosity coefficient $\eta_{\|}(z)$ depends on the transverse position in the film $z$. Since equation (61) involves only the lateral components of the stress, the effective shear viscosity $\tilde{\eta}$ is obtained by averaging Eq. (61) across the film. Using notation (2) we thus get

$$
\tilde{\eta}=h \bar{\eta}_{\|},
$$

which follows from expressions (48), (49), (A7), and A13).

\section{Expansion viscosity of the film}

The effective expansion viscosity $\tilde{\kappa}$ is obtained by evaluating the stress response of the film to the axisymmetric straining flow with the strain-rate tensor given by

$$
E=\left[\begin{array}{rrr}
1 & 0 & 0 \\
0 & 1 & 0 \\
0 & 0 & -2
\end{array}\right]
$$

which corresponds to a uniform expansion of the film. By axial symmetry, the $3 \mathrm{D}$ viscous-stress tensor can be expressed as a combination of the normal and lateral terms,

$$
\boldsymbol{\tau}^{\prime}=\kappa_{\perp} \hat{\mathbf{e}}_{z} \hat{\mathbf{e}}_{z}+\kappa_{\|}(z) \mathbf{I}_{\mathrm{s}},
$$


$(a)$

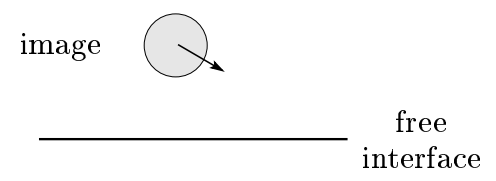

particle

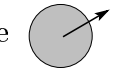

fluid

(b)

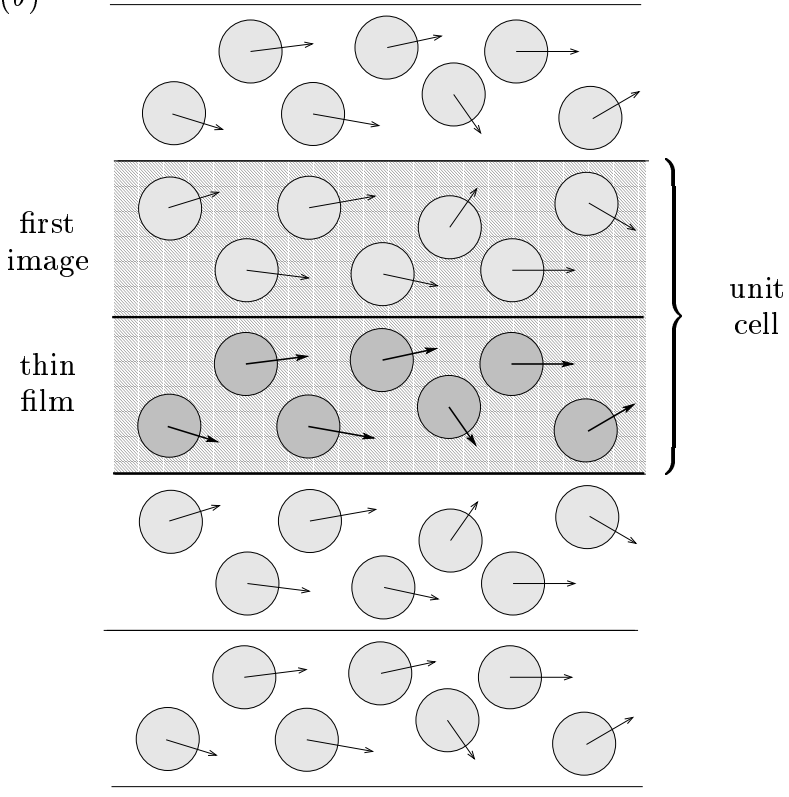

FIG. 6: Reflection method: (a) single free interface; (b) representation of a particle-stabilized film in terms of an unbounded periodic system.

where $\kappa_{\perp}$ is independent of $z$, owing to the continuity of the transverse momentum flux. Integrating the above relation with respect to $z$, and recalling that the effective viscous stress is associated with the excess part (A13) of the stress tensor, we find

$$
\tilde{\kappa}=h\left(\bar{\kappa}_{\|}-\kappa_{\perp}\right) .
$$

In our numerical calculations, the deviatoric part of the average stress tensor (64) is determined directly from the stresslet component of the induced-force distribution on the particle surfaces. To obtain the relation that links $\tilde{\kappa}$ to our numerical results, we rewrite equation (64), averaged across the film, in the form

$$
\overline{\boldsymbol{\tau}}^{\prime}=\bar{\alpha} \mathbf{I}+\bar{\beta} \mathbf{E},
$$

where

$$
\bar{\alpha}=\frac{1}{3}\left(\kappa_{\perp}+2 \bar{\kappa}_{\|}\right), \quad \bar{\beta}=\frac{1}{3}\left(\bar{\kappa}_{\|}-\kappa_{\perp}\right),
$$

and $E$ is the strain-rate tensor (63). Comparing relations (65) and (67), we find that the expansion film viscosity and the amplitude $\bar{\beta}$ of the deviatoric part of the average stress (66) are related by equation

$$
\tilde{\kappa}=3 h \bar{\beta} .
$$

\section{Particle mobility}

The collective mobility coefficient (58) was determined by evaluating the particle flux $\tilde{\mathbf{j}}_{\mathrm{c}}$ produced by a constant lateral external force (11b) in a uniform film with zero net force (12) and zero net volume flow. The zero-net-force condition and the definition (36) of the excess potential (i.e., potential corrected for the buoyancy contribution) imply that the excess force and total force acting on the colloidal particles are directly related,

$$
\nabla_{\mathrm{s}} \Psi_{\mathrm{c}}^{\prime}=\left(1-\phi_{\mathrm{c}}\right) \nabla_{\mathrm{s}} \Psi_{\mathrm{c}}
$$

Thus, for a uniform system, Eq. (57) can be rewritten as the mobility relation

$$
\tilde{\mathbf{j}}_{\mathrm{c}}=-\tilde{n}_{\mathrm{c}} \nu \nabla_{\mathrm{s}} \Psi_{\mathrm{c}}
$$

where

$$
\nu=\left(1-\phi_{\mathrm{c}}\right) \nu^{\prime}
$$

is the mobility coefficient describing the response of the system to the total (rather than excess) force. For compatibility with the usual definition of the collective mobility in bulk suspensions, we present our results for the coefficient $\nu$ instead of $\nu^{\prime}$.

\section{B. Reflection method}

To obtain the shear and expansion film-viscosity coefficients $\tilde{\eta}$ and $\tilde{\kappa}$, the stress response of the film with a constant thickness $h$ to the flow fields (60) and (63) has to be evaluated. Similarly, the collective mobility coefficient $\nu$ is obtained by calculating the particle flux produced by a constant lateral force acting on the particles. To solve these hydrodynamic problems we use a standard Stokesian-dynamics algorithm for free space, combined with a flow reflection method.

Our reflection technique is based on the well-known observation that the flow reflected from a free interface at $z=0$ has the form

$$
\mathbf{v}^{\text {out }}(x, y, z)=\left(\mathrm{I}_{\mathrm{s}}-\hat{\mathbf{e}}_{z} \hat{\mathbf{e}}_{z}\right) \cdot \mathbf{v}^{\text {in }}(x, y,-z),
$$

where $\mathbf{v}^{\text {in }}$ is the incoming flow. Accordingly, the image of a spherical particle centered at $(X, Y, Z)$ and moving with the velocity $\left(U_{x}, U_{y}, U_{z}\right)$ is a sphere centered at $(X, Y,-Z)$, moving with the velocity $\left(U_{x}, U_{y},-U_{z}\right)$. This reflection geometry is illustrated in Fig. 6( $a)$. 
To obtain the solution for particle motion in a film with periodic boundary conditions in the lateral directions, we use a Stokesian-dynamics algorithm for a periodic system of particles in free space. We construct an elementary cell in $3 \mathrm{D}$ from an elementary cell in the film and the reflection of this cell in the upper interface, as illustrated in Fig. 6(b). Multiple reflections of the composed elementary cell in the 3D system ensure that the free-boundary boundary conditions are satisfied both on the lower and the upper film interface. Thus, the periodic 3D system and the doubly periodic liquid film with free interfaces are hydrodynamically equivalent.

We note, however, that our hydrodynamic calculations in the thin-film geometry differ from the usual results for $3 \mathrm{D}$ periodic systems. Namely, in the thin-film case, the spheres are not allowed to overlap the lateral planes separating periodic cells, because these planes represent the film interfaces. This constraint is not required in simulations of suspensions in an unbounded space.

In our numerical calculations we use the hydrodynamic-interactions algorithm introduced in [23], with further improvements described in [24]. The algorithm combines a multipolar expansion of the flow field in the system, with a superposition of two-particle lubrication corrections. Our algorithm includes the multipolar expansion of Stokes flow to an arbitrary order, thus accurate results, with controlled multipolar-truncation error, can be obtained.

\section{Simulation details}

The short-time film viscosity coefficients $\tilde{\eta}$ and $\tilde{\kappa}$ were determined using relation

$$
\overline{\boldsymbol{\tau}}^{\prime}=V^{-1} \sum_{i=1}^{N} \mathcal{S}_{i}
$$

between the average stress tensor (62) or (66) and the stresslets

$$
\mathcal{S}_{i}=\frac{1}{2} \int\left[\mathbf{r}_{i} \mathbf{F}_{i}\left(\mathbf{r}_{i}\right)+\mathbf{F}_{i}\left(\mathbf{r}_{i}\right) \mathbf{r}_{i}-\frac{2}{3} \mathbf{r}_{i} \cdot \mathbf{F}_{i}\left(\mathbf{r}_{i}\right) \mathbf{l}\right] d \mathbf{r}_{i}
$$

of the induced-force distribution $\mathbf{F}_{i}$ on the surfaces of particles $i=1, \ldots, N$ (where $\mathbf{r}_{i}=\mathbf{r}-\mathbf{R}_{i}$ is the relative position vector with respect to the center of particle $i)$. The film-viscosity coefficients $\tilde{\eta}$ and $\tilde{\kappa}$ were evaluated using relations (62) and (68) for a suspension of spheres in the external 2D straining flow (60) and axisymmetric straining (63) flow, respectively. The collective lateral particle mobility was obtained from the relation

$$
\nu=\frac{1}{2} N^{-1} \sum_{i, j=1}^{N} \boldsymbol{\mu}_{i j}^{\mathrm{tt}}: \mathrm{I}_{\mathrm{s}}
$$

where $\boldsymbol{\mu}_{i j}^{\mathrm{tt}}$ is the translational component of the manyparticle translational mobility matrix in a periodic system with zero net flow.
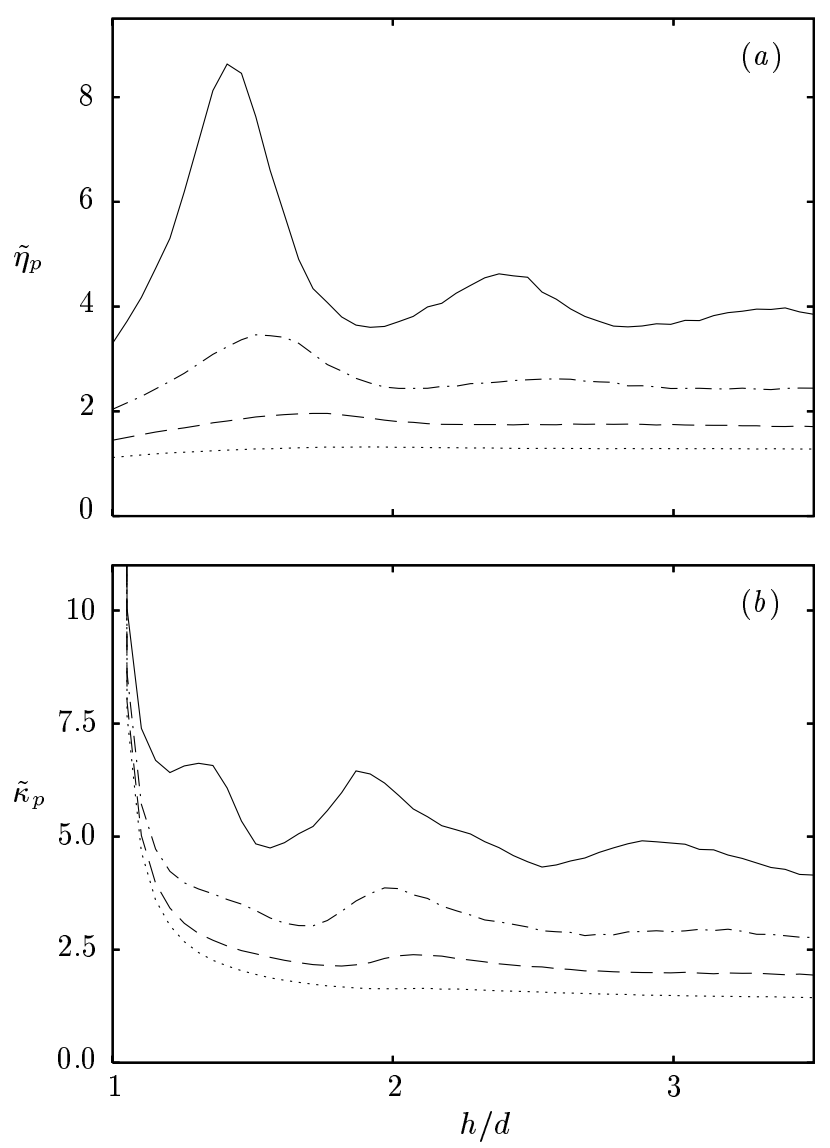

FIG. 7: Normalized particle contributions to the $(a)$ shear and $(b)$ expansion film-viscosity coefficients, as defined by Eqs. (76), versus film thickness $h$, for particle volume fractions $\phi_{\mathrm{c}}=0.1$ (dotted line), 0.2 (dashed), 0.3 (dash-doted), 0.4 (solid).

The quantities (73)-(75) were determined for an equilibrium distribution of $N=40,80$ and 160 spheres in films of different thickness $h$. The results were averaged over several hundred particle configurations, obtained using a Monte-Carlo method. In our calculations, the force multipoles with the vector-spherical-harmonic orders up to $l=5$ were included.

\section{Numerical results}

Our numerical results for the shear and expansion filmviscosity coefficients $\tilde{\eta}$ and $\tilde{\kappa}$ are depicted in Fig. 7, and those for the collective lateral mobility coefficient $\nu$ are shown in Fig. 8. The film-viscosity coefficients $\tilde{\eta}$ and $\tilde{\kappa}$ are presented in terms of the normalized particle contributions $\tilde{\eta}_{p}$ and $\tilde{\kappa}_{p}$, defined by the relations

$$
\begin{gathered}
\tilde{\eta}=\eta_{0} h\left(1+\frac{5}{2} \phi_{\mathrm{c}} \tilde{\eta}_{p}\right), \\
\tilde{\kappa}=\frac{15}{2} \phi_{\mathrm{c}} \eta_{0} h \tilde{\kappa}_{p},
\end{gathered}
$$




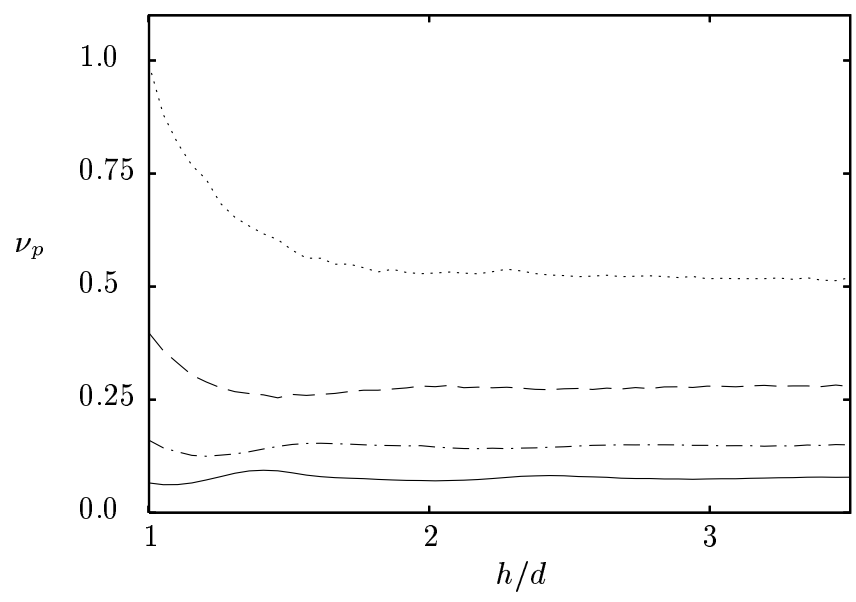

FIG. 8: Normalized collective mobility coefficient, as defined by Eq. (77), versus film thickness $h$, for the same values of volume fraction $\phi_{\mathrm{c}}$ as in Fig. 7

where $\eta_{0}$ is the viscosity of the suspending fluid. Particle mobility in the film is characterized by the dimensionless mobility coefficient $\nu_{p}$, normalized by the mobility of an isolated particle in free space

$$
\nu=3 \pi \eta_{0} d \nu_{p}
$$

With the above definitions, we have $\tilde{\eta}_{p}, \tilde{\kappa}_{p}, \nu_{p} \rightarrow 1$ in the low-density and thick-film limit $\phi_{\mathrm{c}} \rightarrow 0$ and $h / d \rightarrow$ $\infty$. The transport coefficients $\tilde{\eta}_{p}, \tilde{\kappa}_{p}$ and $\nu_{p}$ are plotted in Figs. 7 and 8 versus the film thickness $h$ for several particle volume fractions $\phi_{\mathrm{c}}$.

The results in Fig. 7 indicate that the normalized filmviscosity coefficients depend on the film thickness in an oscillatory way, especially in the high volume-fraction regime. The oscillations are more pronounced for the shear-viscosity coefficient, which has a large peak at $h / d \approx 1.4$ [close to the position of the peak of the lateral pressure $\bar{p}_{\mathrm{c} \|}$, shown in Fig. [2(b)]. At high volume fractions, the normalized mobility coefficient $\nu_{p}$, depicted in Fig. 8, also has significant oscillations. The oscillations are more clearly visible in the plot shown in Fig. 9 $(a)$ on an expanded scale.

According to the results depicted in Fig. 7( $(a)$, the normalized shear viscosity of the film $\tilde{\eta}_{p}$ assumes a minimal value at $h / d=1$. This behavior stems from the fact that for lateral particle motion, the image particles move in the same direction as the original ones (cf. the schematic representation in Fig. (6). Such a collective motion results in a reduced dissipation. For the same reason, the collective mobility $\nu_{p}$ has a maximum at $h / d=1$, as shown in Fig. 8. In contrast, the expansion-viscosity coefficient $\tilde{\kappa}_{p}$, plotted in Fig. $7(b)$, has an $O\left(\epsilon^{-1}\right)$ singularity at $h / d=1$ (where $\epsilon=h / d-1$ denotes a typical gap between the particle surfaces and the film interfaces), owing to the lubrication resistance between the original particles and their images (which have the opposite normal velocity component). At low particle volume fractions

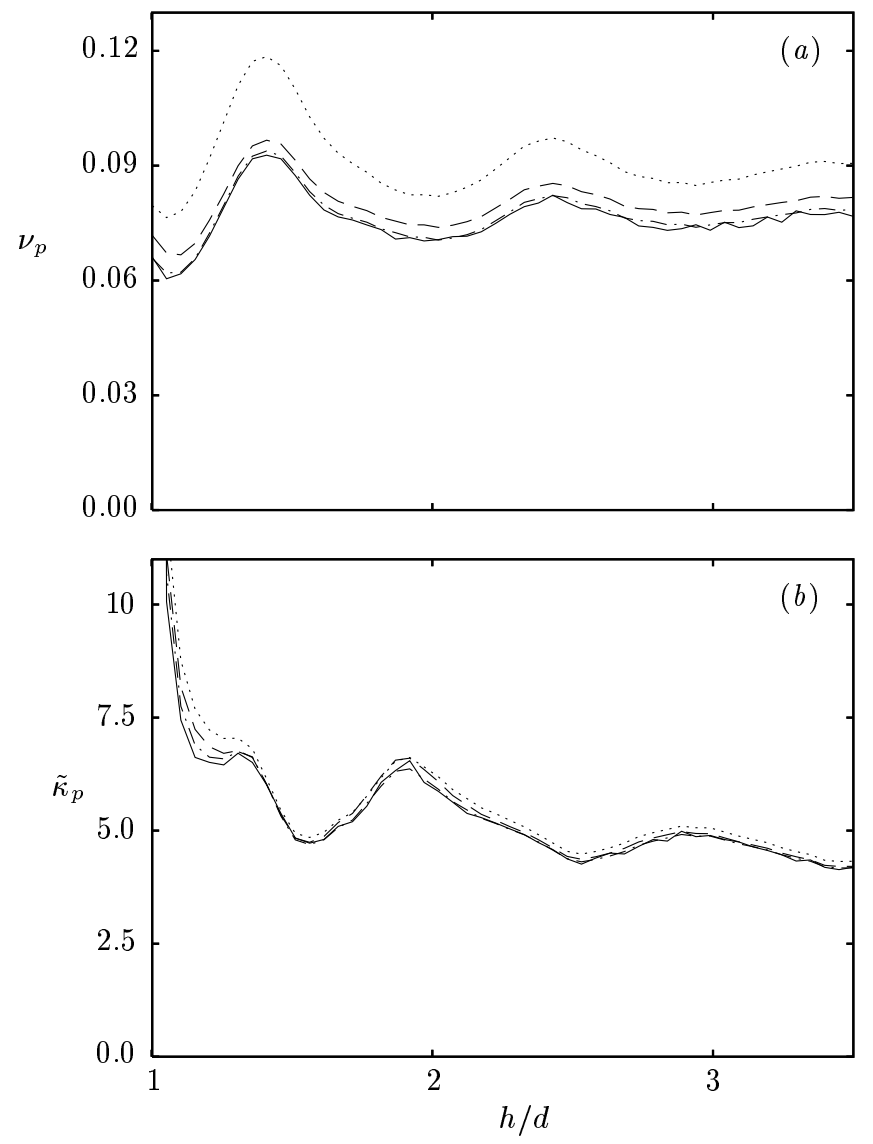

FIG. 9: Normalized (a) collective mobility coefficient and (b) particle contribution to the expansion viscosity of a particlestabilized film, versus film thickness $h$, for $\phi_{\mathrm{c}}=0.4$ and spherical-harmonics truncation order $l_{\max }=2$ (dotted line), 3 (dashed) 4 (dash-dotted), and 5 (solid).

the collective mobility coefficient $\nu_{p}$ diverges as $\log \left(\phi_{\mathrm{c}}\right)$, because of the logarithmic far-field behavior of 2D Stokes flow.

\section{E. Convergence tests}

The results for the film-viscosity and mobility coefficients shown in Figs. 7 and 8 were obtained for a system of $N=80$ particles using induced-force multipoles up to the spherical-harmonics order $l_{\max }=5$. The convergence of the results with $l_{\max }$ is illustrated in Fig. 9 and with the system size $N$ in Fig. 10 for the highest particle volume fraction considered in our simulations, $\phi_{\mathrm{c}}=0.4$.

The results shown in Fig. 9( $a$ ) for the mobility coefficient $\nu$ indicate that the truncation of the multipolarexpansion at the order $l_{\max }=2$ yields an error of $20 \%$ to $30 \%$ for this quantity. The results obtained with $l_{\max }=3$ have the accuracy better than $8 \%$ and $l_{\max }=4$ yields results accurate within $1 \%$. We note that $l_{\max }=2$ includes all multipoles of the STD scheme used by Brady 


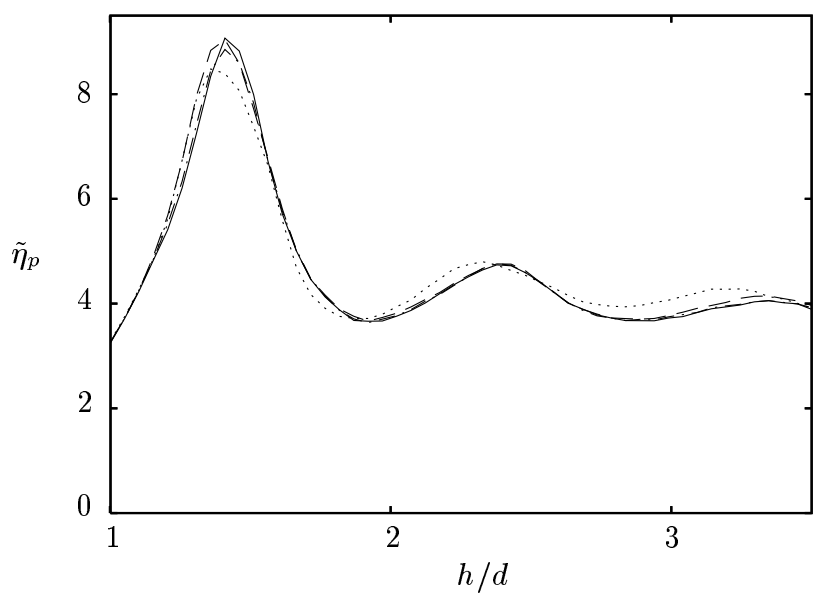

FIG. 10: Normalized particle contributions to the shear viscosity of a particle-stabilized film, versus the film thickness $h$, for $\phi_{\mathrm{c}}=0.4$ and system size $N=20$ particles (dotted line), 40 (dashed), 80 (dashed-dotted), and 160 (solid).

and his collaborators [31], and thirteen other flow multipoles (including potential flows). Thus, for the mobility coefficient $\nu$ the standard STD scheme is insufficient for obtaining accurate results, even if the lubrication corrections are included in the numerical scheme.

Our numerical results for the film-viscosity coefficients $\tilde{\eta}$ and $\tilde{\kappa}$ indicate that the convergence with $l_{\max }$ is faster for these quantities than the convergence for the collective mobility $\nu$. As illustrated in Fig. [9(b), the truncation at $l_{\max }=2$ already yields quite accurate results for the coefficient $\tilde{\kappa}$, except for films with $h / d \lesssim 1.5$. For $\tilde{\eta}$ the convergence with $l_{\max }$ is similar.

The convergence of our results with the system size is illustrated in Fig. 10] where the results for the effective shear-viscosity coefficient $\tilde{\eta}$, evaluated with $l_{\max }=2$, are shown for systems with $N$ between 20 and 160 particles in a unit cell. The results indicate that the convergence of the shear viscosity $\tilde{\eta}$ is fast. A similar convergence has been obtained obtained for the expansion viscosity $\tilde{\kappa}$ and the collective mobility $\nu$.

\section{CONCLUSIONS}

Our unified quasi-two-dimensional description of equilibrium states and transport properties in particlestabilized thin liquid films relies on the film-tension representation of the film thermodynamics. In this representation the system is characterized by extensive parameters corresponding to conserved quantities: the film volume, film area, and the number of fluid molecules and colloidal particles. The conjugate intensive parameters are the normal pressure, film tension, and chemical potentials of the fluid phase and the colloidal component.

Equilibrium conditions and their physical interpretation have been analyzed for nonuniform films in external potentials. In particular, we have shown that the particle contribution to film tension is associated with the excess lateral pressure beyond the uniform isotropic background pressure $p^{(\mathrm{e})}$. Since the normal pressure in the film is equal to the external pressure, the excess lateral pressure equals the difference between the lateral and normal pressure components.

Based on our thermodynamic formalism and the localequilibrium assumption, we have also introduced a quasitwo-dimensional description of transport processes in particle-stabilized liquid films. In some aspects, a film composed of an incompressible suspension is analogous to a $2 \mathrm{D}$ compressible fluid, with the film thickness playing the role of the mass density per unit area, and film tension being similar to the pressure (with a minus sign).

When deviations from equilibrium are small, the momentum transport in the film is characterized by two linear viscosity coefficients: the shear and expansion viscosities. The particle diffusive transport is described by two kinetic coefficients relating the particle flux to the gradients of the normal component of the osmotic pressure $p_{\mathrm{c} \perp}$ and the excess particle chemical potential $\mu_{\mathrm{c}}^{\prime}$. Using the Gibbs-Duhem relation in the film-tension representation, the two kinetic coefficients have been combined into a single collective mobility coefficient. Our explicit calculations of the short-time linear transport coefficients for a film stabilized by a hard-sphere suspension indicate that these coefficients are oscillatory functions of film thickness. The oscillations are due to the short-range layering order in the film.

While the results of our investigations provide a convenient framework for describing equilibrium and nonequilibrium properties of particle-stabilized thin liquid films, there still remain many open questions. First, we considered only films with surfactant-free interfaces. Surfactant adsorbed on the interfaces would contribute to film tension, but otherwise it should not significantly alter the equilibrium conditions in the film. It would, however, substantially influence the relaxation times for decay of nonuniformities of different intensive thermodynamic parameters. Thus partial equilibrium states in stratified films (discussed in [17]) can be significantly affected.

Adsorbed surfactant modifies not only the equilibrium equations of state, but also changes the film viscosity and particle mobility, because of its effect on the boundary conditions at the film interfaces (c.f., our analysis of this problem presented in [32]). The hydrodynamicinteraction algorithm used in the present paper is applicable only to surfactant-free interfaces. However, our recently developed Cartesian-representation method 33, 34, 35]) can be applied to evaluate the effective transport coefficients in a particle-stabilized film with more complex boundary conditions.

Another important problem that has not been addressed here is transport through contact lines between phases of different thickness in stratified films. An analysis in 36 suggests that such transport may control the rate of expansion of thin spots in the film during a 
stepwise-thinning process. On the other hand, hydrodynamic scaling arguments [17] seem to imply that particle transport through a contact line should be faster than particle diffusion (at least in surfactant-free films). This transport, however, can be significantly slowed down, owing to surfactant effects, and because of the entropic barrier associated with a change of particle arrangement at the line separating phases of different thickness. This problem will be analyzed in our future publications.

\section{Acknowledgments}

This work was supported by NSF CAREER grant CTS-0348175; EW was also supported by Polish Ministry of Science grant N501 020 32/1994.

\section{APPENDIX A: EFFECTIVE SURFACE STRESS}

In this appendix we derive the effective lateral stressbalance equation under creeping-flow conditions in a thin film with varying width $h$. The film occupies the region between the lower and upper interfaces

$$
z=z_{\mathrm{L}}(x, y), \quad z=z_{\mathrm{U}}(x, y)
$$

The region occupied by the film can be described by the characteristic function,

$$
\theta_{\mathrm{f}}(\mathbf{r})=\theta\left(z-z_{\mathrm{L}}\right) \theta\left(z_{\mathrm{U}}-z\right)
$$

where $\theta$ denotes the Heaviside step function.

The film is surrounded by a gas with a constant pressure $p^{(\mathrm{e})}$, and a lateral force field

$$
\mathbf{f}(\mathbf{r})=\theta_{\mathrm{f}}(\mathbf{r}) \mathbf{f}(\mathbf{r})
$$

acts on the film. Accordingly, the stress tensor in the whole $3 \mathrm{D}$ space

$$
\boldsymbol{\tau}_{\mathrm{tot}}=-\left(1-\theta_{\mathrm{f}}\right) p^{(\mathrm{e})} \mathbf{I}+\theta_{\mathrm{f}} \boldsymbol{\tau}
$$

(where $\tau$ denotes the stress tensor in the film, and $p^{(\mathrm{e})}$ is the external pressure) satisfies the full 3D balance equation

$$
\nabla \cdot \tau_{\text {tot }}=-\mathbf{f}
$$

There are no capillary-pressure and Marangoni-stress contributions in the above equation, because of our assumptions that the curvature of the interfaces is small, and the interfacial tension is constant. If needed, such contributions can be added as appropriate delta distributions of the force on the film interfaces.
To obtain the film-tension representation for the lateral stress balance in the film, it is convenient to reorder terms in Eq. (A4),

$$
\boldsymbol{\tau}_{\text {tot }}=-p^{(\mathrm{e})} \mathbf{I}+\theta_{\mathrm{f}} \delta \boldsymbol{\tau} .
$$

The first term on the right-hand side of this equation is the constant background pressure that does not contribute to the stress-balance (A5). The second term represents the excess stress in the film. Using the continuity of the transverse momentum flux and the associated boundary condition (51) for the normal stress we find

$$
\delta \boldsymbol{\tau}=\left(\tau_{\|}-\tau_{\perp}\right) \mathrm{I}_{\mathrm{s}}
$$

By inserting (A6) into the stress-balance equation (A5 and integrating with respect to the variable $z$, we obtain

$$
\int_{\infty}^{\infty} d z\left(\hat{\mathbf{e}}_{z} \frac{\partial}{\partial z}+\nabla_{\mathrm{s}}\right) \cdot \theta_{\mathrm{f}} \delta \boldsymbol{\tau}=-\tilde{\mathbf{f}}
$$

where $\tilde{\mathbf{f}}$ is the force density per unit area of the film. The first term on the left-hand side of the above relation vanishes because $\theta_{\mathrm{f}}(x, y, z)=0$ for $z= \pm \infty$. In the second term we change the order of integration and differentiation to obtain the quasi-two-dimensional stress-balance equation for the excess stress,

$$
\nabla_{\mathrm{s}} \cdot h \delta \overline{\boldsymbol{\tau}}=-\tilde{\mathbf{f}}
$$

where the bar denotes the average (2).

In an equilibrium state, only the equilibrium pressure tensor (11) contributes to $\tau$,

$$
\boldsymbol{\tau}=-\left(p_{\perp} \hat{\mathbf{e}}_{z} \hat{\mathbf{e}}_{z}+p_{\|} \mathbf{l}_{\mathrm{s}}\right)
$$

Taking into account the boundary condition (7) and the definition of film tension (5), we find that relations (A9) and (A10) yield the lateral stress balance (17).

In a more general, nonequilibrium case, Eq. (A9) is split into the lateral and vertical components,

$$
\begin{gathered}
\nabla_{\mathrm{s}} \cdot h \delta \overline{\boldsymbol{\tau}} \cdot \mathrm{I}_{\mathrm{s}}=-\tilde{\mathbf{f}} . \\
\boldsymbol{\nabla}_{\mathrm{s}} \cdot h \delta \overline{\boldsymbol{\tau}} \cdot \hat{\mathbf{e}}_{z}=0,
\end{gathered}
$$

Since the normal component A12 vanishes on the assumption that there is no transverse external force, we find that (A11) yields the lateral stress balance (47), with the effective $2 \mathrm{D}$ stress

$$
\tilde{\tau}=h \delta \bar{\tau} \cdot I_{\mathrm{s}}
$$

[1] V. Bergeron, Curr. Opin. Colloid Interface Sci. 4, 249 (1999).
[2] C. Stubenrauch and R. von Klitzing, J. Phys.: Condens. 
Matter 15, R1197 (2003).

[3] P. A. Kralchevsky, K. D. Danov, and I. B. Ivanov, in Foams. Theory, Measurements, and Applications, edited by R. K. Prud'homme and S. A. Khan (Dekker, New York, 1996), pp. 1-98.

[4] J. Czarnecki, S. D. Taylor, and J. Masliyah, J. Colloid Interface Sci. 282, 499 (2005).

[5] A. D. Nikolov, D. T. Wasan, P. A. Kralchevsky, and I. B. Ivanov, Colloids Surf. 67, 139 (1992).

[6] A. D. Nikolov and D. T. Wasan, Langmuir 8, 2985 (1992).

[7] E. S. Basheva, K. D. Danov, and P. A. Kralchevsky, Langmuir 13, 4342 (1997).

[8] A. Henderson, D.and Trokhymchuk, A. Nikolov, and D. T. Wasan, Ind. Eng. Chem. Res. 44, 1175 (2005).

[9] V. Bergeron and C. J. Radke, Langmuir 8, 3020 (1992).

[10] O. Théodoly, J. S. Tan, R. Ober, C. E. Williams, and V. Bergeron, Langmuir 17, 4910 (2001).

[11] P. Tchoukov, E. Mileva, and D. Exerova, Colloids Surf. A 238, 19 (2004).

[12] Y. Horiuchi, H. Matsumara, and K. Furusawa, J. Colloid Interface Sci. 207, 41 (1998).

[13] J. Czarnecki, private communication.

[14] E. S. Basheva, P. A. Kralchevsky, K. D. Danov, K. P. Ananthapadmanabhan, and A. Lips, Phys. Chem. Chem. Phys. 9, 5183 (2007).

[15] G. N. Sethumadhavan, A. D. Nikolov, and D. Wasan, Langmuir 17, 2059 (2001).

[16] S. Sethumadhavan, Bindal, G. N., A. D. Nikolov, and D. T. Wasan, Colloids Surfaces A: Physicochem. Eng. Aspects 204, 51 (2002).

[17] J. Bławzdziewicz and E. Wajnryb, Europhys. Lett. 71, 269 (2005).

[18] X. L. Chu, A. D. Nikolov, and D. T. Wasan, J. Chem. Phys. 103, 6653 (1995), erratum in: J. Chem. Phys. 105 4892 (1996).

[19] A. Trokhymchuk, D. Henderson, A. D. Nikolov, and D. T. Wasan, Langmuir 17, 4940 (2001).

[20] A. Trokhymchuk, D. Henderson, A. Nikolov, and D. T. Wasan, J. Phys. Chem. B 107, 3927 (2003).

[21] For earlier discussions of thin-film thermodynamics see 2, 3, 22] and references therein.

[22] N. Ikeda, R. Krustev, and H.-J. Müller, Adv. Colloid
Interface Sci. 108-9, 273 (2004).

[23] B. Cichocki, B. U. Felderhof, K. Hinsen, E. Wajnryb, and J. Bławzdziewicz, J. Chem. Phys. 100, 3780 (1994).

[24] M. L. Ekiel-Jeżewska and E. Wajnryb, in Theoretical Methods for Micro Scale Viscous Flows, edited by F. Feuillebois and A. Sellier (Research Signpost, in press).

[25] H. B. Callen, Thermodynamics and an Introduction to Thermostatistics (Wiley, New York, 1985).

[26] J. G. Kirkwood and F. Buff, J. Chem. Phys. 17, 338 (1949).

[27] Momentum transfer in suspensions is mediated through the suspending fluid. However, equilibrium properties of a suspension of hard spheres in an inert incompressible medium are equivalent to those of a fluid of hard spheres undergoing Newtonian dynamics in empty space. Therefore, in equilibrium considerations we can entirely neglect the effect of the suspending fluid.

[28] In our previous paper [17], we plotted the modified film tension (40) rather than (27). However, since the quantities $\gamma_{\mathrm{c}}$ and $\hat{\gamma}_{\mathrm{c}}$ differ only by the normal pressure multiplied by a constant factor [cf. Eq. [41)], the equilibrium conditions $\gamma_{\mathrm{c}}=$ const and $p_{\mathrm{c} \perp}=$ const imply that the modified film tension $\hat{\gamma}_{\mathrm{c}}$ is also constant in the film.

[29] T. K. Vanderlick, L. E. Scriven, and H. T. Davis, J. Chem. Phys. 90, 2422 (1989).

[30] P. Mazur and S. R. de Groot, Non-Equilibrium Thermodynamics (Dover, 1984).

[31] J. F. Brady and G. Bossis, Ann. Rev. Fluid Mech. 20, 111 (1988).

[32] J. Bławzdziewicz, V. Cristini, and M. Loewenberg, Phys. Fluids 11, 251 (1999).

[33] S. Bhattacharya, J. Bławzdziewicz, and E. Wajnryb, Physica A 356, 294 (2005).

[34] S. Bhattacharya, J. Bławzdziewicz, and E. Wajnryb, J. Fluid Mech. 541, 263 (2005).

[35] S. Bhattacharya, J. Bławzdziewicz, and E. Wajnryb, J. Comput. Phys. 212, 718 (2006).

[36] P. A. Kralchevsky, A. D. Nikolov, D. T. Wasan, and I. B. Ivanov, Langmuir 6, 1180 (1990). 